\title{
Special issue on recommender system
}

\author{
Bin Guo ${ }^{1} \cdot$ Xing Xie ${ }^{2} \cdot{\text { Lina } \mathrm{Yao}^{3} \cdot \text { Yong } \mathrm{Li}^{4} \cdot \text { Cecilia Mascolo }}^{5} \cdot \mathrm{Xia} \mathrm{Hu}^{6}$
}

Published online: 25 November 2019

(c) China Computer Federation (CCF) 2019

\section{Introduction}

Intelligent recommendation is to deliver personalized services and items to users. Recommender systems are utilized in various areas such as movies, music, news, posts, POIs, and products. With the rapid development of social media and intelligent sensing techniques, we can now gather huge amount of user-contributed data, which can be leveraged to better predict personality traits and draw user profiles. However, the cross-space, multi-modal, and data sparsity nature also raises numerous challenges for recommendation. In recent years, the surging of deep learning techniques provides new opportunities for dealing with such issues. Numerous new models, such as neural collaborative filtering, Wide and Deep, DeepFM, xDeepFM, are thus raised. Several new directions such as explainable recommendation, recommendation with knowledge graph are also being investigated. This theme issue aims to provide an opportunity for researchers and product developers to review and discuss the

Bin Guo

guob@nwpu.edu.cn

Xing Xie

xie.xing@microsoft.com

Lina Yao

lina.yao@unsw.edu.au

Yong Li

liyong07@tsinghua.edu.cn

Cecilia Mascolo

cm542@cam.ac.uk

$\mathrm{Xia} \mathrm{Hu}$

hu@cse.tamu.edu

Northwestern Polytechnical University, Xian, China

2 Microsoft Research Asia, Bejing, China

3 The University of New South Wales, Sydney, Australia

4 Tsinghua University, Bejing, China

5 Cambridge University, Cambridge, UK

6 Texas A and M University, College Station, USA state-of-the-art and trends of recommendation techniques and applications.

\section{In this issue}

The articles in this issue highlight relevant current technologies and approaches pertaining to recommender systems, including theoretical studies, practical issues, emerging technologies and innovative applications. Over the numerous submissions, we have finally accepted five high quality papers. The selected papers fall into two main research topics of recommender systems: three are about recommendation systems in particular areas (news, ride-sharing, and job), and two about general recommendation algorithms.

The first article, "An efficient ride-sharing recommendation for maximizing acceptance on geo-social data" by Tang et al. describes a novel approach that uses heterogeneous information network to maximize people's acceptance for ridesharing. The observed geographic proximity is usually treated as a clue for grouping riders, however, trust between unacquainted riders when sharing a trip is often overlooked. Tang leverages existing spatiotemporal trajectories organized as a meta-path to acquire a vast set of driver-rider pairs. With underlying trust links between riders in each pair, the rider's willingness for joining in a group is measured using social context, and subsequently, a ridesharing group is established by reaching the best compromise between user satisfactory and recommendation quality.

The second article, "NCR-KG: News Community Recommendation with Knowledge Graph" by Bai et al. describes a news recommendation framework that uses knowledge graph to represent each user's profile, including the personal information and tags that are extracted from historical clicked records. Users are clustered with a new similarity computation method, which generates users community. The news are recommended collaboratively with the records of all users in the community. As for the timeliness of news, an update method is proposed for the latest incoming news. The experiments are conducted with metrics evaluation and 
manual evaluation, and the results demonstrate the effectiveness and efficiency of the proposed framework.

The third article "Job Recommendation Algorithm for Graduates Based on Personalized Preference" by Zhou et al. describes a job recommendation algorithm for graduates considering their characteristics and personalized preference for jobs. Collaborative filtering method is currently the most frequently adopted and effective recommendation algorithm, but it cannot be directly applied to job recommendation for graduates because they generally have no historical records on employment. To solve this problem, massive campus records are used for user clustering and user modeling. The job recommendation algorithm takes account of both the group records of job choices and the individual preferences for jobs.

The fourth article, "Learning from Visual Content and Style: An Image-enhanced Recommendation Model" by Luo et al. proposes a deep learning method for recommending images that suit users' interests. Based on the state-of-the-art Visual Bayesian Personalized Ranking (VBPR) model for visual recommendation, the authors study the problem of learning both the visual content and style for image recommendation. They leverage advanced methods in computer vision to learn the visual content and style representation, and study how to combine visual signals with users' collaborative data. Experimental results show the effectiveness of the proposed model compared to existing methods.

The fifth article, "Helpfulness-aware Review based Neural Recommendation" by Ge et al. presents a neural network based recommendation method with the support of helpful reviews, which contain rich information of user interests and item characteristics. Different from existing review-based recommendation methods that only utilize the content of reviews while ignoring the helpfulness scores, the paper incorporates both of them in recommendation. The approach consists of a review encoder and a user/item encoder. Since many reviews do not have helpfulness scores, they propose a neural helpfulness prediction model to predict the helpfulness scores of these reviews. Experiment results indicate that the proposed approach outperforms other review-based recommendation methods.

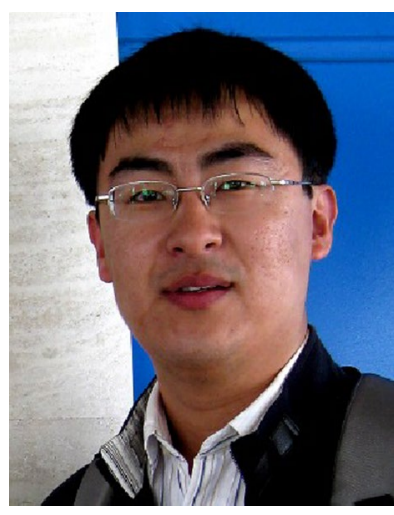

Bin Guo is a professor from Northwestern Polytechnical University, China. He received his $\mathrm{Ph} . \mathrm{D}$. degree in computer science from Keio University, Tokyo, Japan, in 2009. During 2009-2011, he was a post-doctoral researcher at Institute TELECOM SudParis in France. His research interests include pervasive computing, mobile social networking, and mobile crowd sensing. Dr. Guo has served as an associate editor of IEEE
Communications Magazine, IEEE Transactions on Human Machine Systems, and ACM IMWUT.

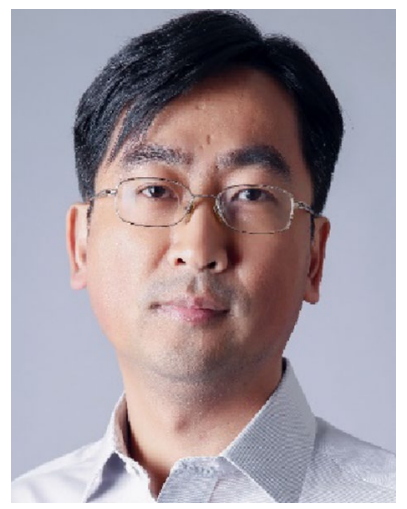

Xing Xie is currently a senior principal research manager at Microsoft Research Asia, and a guest Ph.D. advisor at the University of Science and Technology of China. He received his B.S. and Ph.D. degrees in Computer Science from the University of Science and Technology of China in 1996 and 2001, respectively. He joined Microsoft Research Asia in July 2001, working on data mining, social computing and ubiquitous computing. During the past years, he has published over 300 referred journal and conference papers, won the best student paper award in KDD 2016, and the best paper awards in ICDM 2013 and UIC 2010. He currently serves on the editorial boards of ACM Transactions on Social Computing (TSC), ACM Transactions on Intelligent Systems and Technology (TIST), and so on.

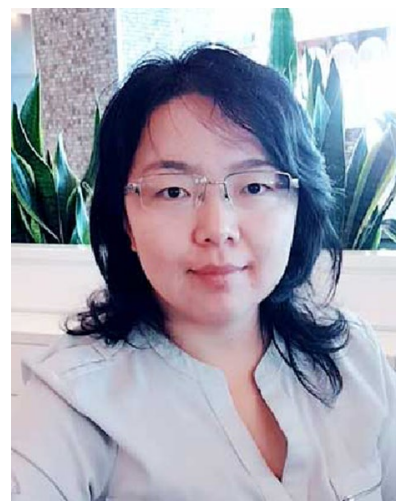

Lina Yao is currently a Senior Lecturer@School of Computer Science and Engineering, The University of New South Wales (UNSW), Australia. She is directing the Data Dynamics Lab (D2 Lab). Her research interest lies in Data Mining and Machine Learning applications with the focuses on recommender systems, human activity recognition, human computer cooperations, Brain Computer Interface and Internet of Things.

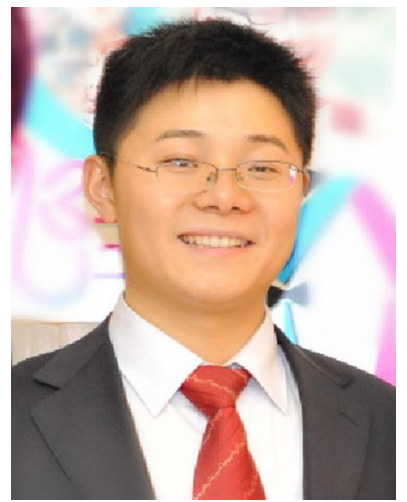

Yong $\mathrm{Li}$ received the B.S. degree from Huazhong University of Science and Technology in 2007, and the M. S. and the Ph. D. degrees in Electrical Engineering from Tsinghua University, in 2009 and 2012, respectively. During 2012 and 2013, he was a Visiting Research Associate with Telekom Innovation Laboratories and Hong Kong University of Science and Technology respectively. During 2013 to 2014, he was a Visiting Scientist with the University of Miami. Currently, he is a Faculty Member of the Department of Electronic Engineering, Tsinghua University. His research interests are in the areas of big data, mobile computing, wireless communications and networking. 


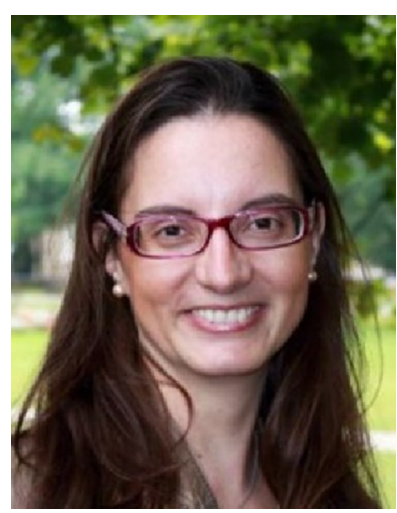

Cecilia Mascolo is a Full Professor of Mobile Systems in the Department of Computer Science and Technology and centre co-director for the Centre for Mobile, Wearable Systems and Augmented Intelligence. My interests are in the study of mobile systems, the learning from their data offline and on device and their applications, especially in terms of mobile health.

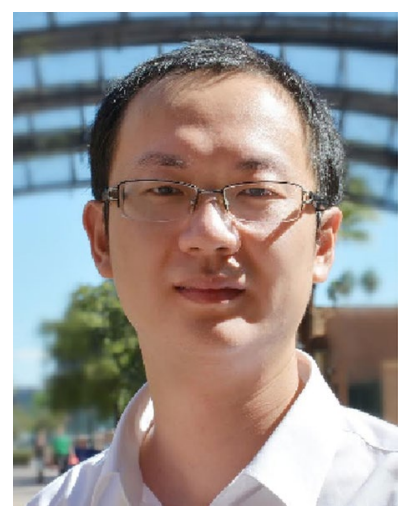

Xia Hu an Assistant Professor in Computer Science and Engineering at Texas A\&M University starting from Fall 2015, and is also a member of the Center for Remote Health Technologies and Systems and the Center for the Study of Digital Libraries. He is currently directing the DATA (Data Analytics at Texas A\&M) Lab. His research interest include automated and interpretable data mining and machine learning algorithms. 